\title{
A BANACH LATTICE NOT WEAKLY PROJECTABLE
}

\author{
E. STRZELECKI
}

(Received 1 June 1971)

Communicated by B. Mond

In [4] a concept of a weakly projectable vector lattice has been introduced. Stone vector lattices [3] and thus all special types of them, like Riesz [5], $\sigma$-complete and complete vector lattices are weakly projectable. Moreover $C[0,1]$ is weakly projectable but not Stone [4]. As we see the collection $W$ of weakly projectable vector lattices is quite large. This explains to some extent the difficulty in producing examples of vector lattices which do not belong to $W$. In this note an example of a Banach lattice [1] which is not weakly projectable is described.

Definition. A vector lattice $E$ is said to be weakly projectable if for any $x, y \in E$ there exists $z \in x^{\perp}$ such that $y \in(|x|+|z|)^{\perp \perp}$.

(For definitions of symbols used above we refer e.g. to [2]).

EXAMPLE. Let $F[0,1]$ denote the space of bounded real valued functions defined on $[0,1]$ and discontinuous at most at a countable set of points. Addition and multiplication by scalars are introduced in the usual way. The order is defined by: $x \geqq 0$ if and only if $x(t) \geqq 0$ for each $t \in[0,1]$. Define also $\|x\|=\sup _{0 \leqq t \leqq 1}|x(t)|$.

Using standard methods it is easy to prove that $F[0,1]$ is a Banach lattice and thus an Archimedean vector lattice [1]. We shall prove that $F[0,1] \notin W$.

Let $\left\{r_{n}\right\}$ be a sequence dense in the interval $[0,1]$. Denote by $A$ the set

$$
A=[0,1] \cap\left(\bigcup_{n=1}^{\infty}\left(r_{n}-4^{-n}, r_{n}+4^{-n}\right)\right) .
$$

$A$ is open in $[0,1]$ and mes $(A) \leqq \frac{2}{3}$, thus $A^{\prime}=[0,1] \backslash A$ is a closed uncountable set. We have also $\bar{A}=[0,1]$. Define $x:[0,1] \rightarrow R$ by

$$
x(t)=\text { distance from } t \text { to } A^{\prime} .
$$

$x$ is continuous on $[0,1]$, and so $x \in F[0,1]$. To show that $F[0,1] \notin W$ it is sufficient to prove that for any $z \in x^{\perp}$, the identity function $e: e(t)=1$ for all $t \in[0,1]$ does not belong to $(|x|+|z|)^{\perp \perp}$. Take any $z \in x^{\perp}$. Then $z(t)=0$ for all $t \in A$. 
Moreover, since $A$ is dense in $[0,1]$, any point $t_{0}$ of $[0,1]$ is a limit of a sequence $\left\{t_{n}\right\}$ of points in $A$. Therefore if $z$ is continuous at $t_{0}$ then $z\left(t_{0}\right)=\lim _{n \rightarrow \infty}$ $z\left(t_{n}\right)=0$. Since $z \in F[0,1]$, it is discontinuous at most at a countable set. On the other hand $A^{\prime}$ is not countable. Consequently, there exists a point $\tau \in A^{\prime}$ such that $z(\tau)=0$. Since $\tau \in A^{\prime}$, we have also $x(\tau)=0$. Thus $w=|z|+|x|$ vanishes at $\tau$. Hence the function $u$ defined by

$$
u(t)= \begin{cases}1 & \text { if } t=\tau \\ 0 & \text { if } t \neq \tau\end{cases}
$$

belongs to $F[0,1]$ and $u \perp w$.

Let $v \in w^{\perp \perp}$. Since $u \in w^{\perp}$ and $u(\tau) \neq 0$, it follows that $v(\tau)=0$. On the other hand $e(\tau)=1$ and thus $e \notin w^{\perp \perp}$. This concludes the proof that $F[0,1]$ is not weakly projectable.

\section{References}

[1] G. Birkhoff, Lattice theory (American Mathematical Society, Providence 1967, Colloquium publication 25).

[2] A. L. Peressini, Ordered topological vector spaces (Harper's Series in Modern Mathematics, 1967).

[3] T. P. Speed, 'On commutative $l$-groups', (to appear)

[4] G. T. Spirason and E. Strzelecki 'A note on $P_{t}$-ideals', J. Austral. Math. Soc., 14 (1972), 304-310.

[5] B. Z. Vulikh, Introduction to the theory of partially ordered spaces. (Wolters-Noordhoff Scientific Publications, Groningen, 1967).

Department of Mathematics

Monash University

Victoria 3168

Australia 